CONFORMAL GEOMETRY AND DYNAMICS

An Electronic Journal of the American Mathematical Society

Volume 3, Pages 37-49 (March 25, 1999)

S 1088-4173(99)00043-0

\title{
TAIMANOV'S SURFACE EVOLUTION AND BÄCKLUND TRANSFORMATIONS FOR CURVES
}

\author{
OSCAR GARAY AND JOEL LANGER
}

\begin{abstract}
Taimanov's evolution of conformally parametrized surfaces in Euclidean space by the modified Novikov-Veselov equation is interpreted here (in the revolution case) using hyperbolic geometry and Bäcklund transformations for curves.
\end{abstract}

\section{INTRODUCTION}

Taimanov [Ta 1, Ta 2] recently studied an interesting evolution for conformally parametrized surfaces in $E^{3}$ (based on an observation of Konopelchenko [Ko]). The evolving surface $\Sigma$ is given by a generalized Weierstrass representation involving a Dirac operator with potential function $U(x, y, t)$ satisfying the modified NovikovVeselov equation (mNV). The evolution turns out to preserve conformal type of $\Sigma$ as well as the Willmore (squared mean curvature) integral, and an infinite sequence of related higher order functionals - the constants of motion for the soliton equation $\mathrm{mNV}$. In the special case of a surface of revolution, $\Sigma$ is described by a planar generating curve $\sigma(x, t)$, and the Novikov-Veselov equation reduces to the modified Korteweg-de Vries equation $(\mathrm{mKdV})$ for $q(x, t)=4 U$.

Here we interpret some of Taimanov's results (in the revolution case) by viewing $\sigma$ as a curve in the upper half-plane model of the hyperbolic plane $H^{2}$, with real axis coinciding with the rotation axis of $\Sigma$. Under Taimanov's evolution (TE), $\sigma$ may then be regarded as a unit speed curve with geodesic curvature $\kappa$, unit tangent $T$, and normal $N$, satisfying the curve evolution equation

$$
\sigma_{t}=\left(\frac{\kappa^{2}}{2}+1\right) T+\kappa_{x} N
$$

Further, $\kappa$ satisfies $\mathrm{mKdV}$, and is a Bäcklund transform of the potential $q$. It follows at once from (CE) that closure of $\sigma$ is preserved (one of Taimanov's results, not obvious from the non-local Weierstrass representation itself); the length of $\sigma$, which determines the conformal type of $\Sigma$, is thus preserved as well. The total squared geodesic curvature of $\sigma$, known to be proportional to the Willmore integral of $\Sigma$, is preserved as constant of motion for mKdV. Such results are proved in Section 3.

To add geometric meaning to the transformation $q \mapsto \kappa$, we consider (static) Bäcklund transformations (BT) for curves $\sigma$ in the hyperbolic plane, and for constant torsion curves $\gamma$ in Lorentzian 3-space; the two are related by viewing the

Received by the editors October 28, 1998.

1991 Mathematics Subject Classification. Primary 35Q51, 35Q53, 53A05, 53A35, 53A30.

We wish to acknowledge the support of the Departamento De Educacion, Universidades E Investigacion, Gobierno Vasco, for J. Langer's visit. 
binormal indicatrix of $\gamma$ as a curve in the hyperboloid model $H^{2} \subset R_{1}^{3}$. The transformations $\gamma \mapsto \tilde{\gamma}$ (BTG), and $\sigma \mapsto \tilde{\sigma}$ (BTB), described in Section 2, are given by vector fields along $\gamma$ (respectively, $\sigma$ ) of constant (Lorentzian) length. Formally, (BTG), may be derived by substituting $i \tau$ for the torsion $\tau$ in the $x$-half of the classical BT for pseudospherical surfaces, restricted to the asymptotic curves (though $q \mapsto \kappa$ is the mKdV, not sine-Gordon BT). As such, (BTG) is based closely on formulas of Calini and Ivey [C-I], who constructed exotic constant torsion knots in $E^{3}$ belonging to the hierarchy of the localized induction equation (to which the Euclidean analogue of (CE) belongs). An interesting (if unsurprising) difference between formulas of [C-I] and the present 'non-compact' case is the appearance of a pair of excluded values of the Bäcklund parameter $C \neq \pm \tau$, corresponding to infinite displacement in (BTG), (BTB). As it turns out, Taimanov's evolution corresponds to precisely the excluded case, so a full geometric description along the above lines necessitates a kind of renormalization at the curve level.

\section{A BÄCKLUND transformation FOR CURVES IN $R_{1}^{3}$}

We consider Lorentzian 3 -space, $R_{1}^{3}$ with metric $\langle\rangle=,\operatorname{diag}(1,1,-1)$. The hyperboloids $\langle V, V\rangle=$ constant $=d$ have constant sectional curvature $K=1 / d$ in the induced (Riemannian or pseudo-Riemannian) metric. In particular, $d<0$ gives (two-copies of) a standard model of the hyperbolic plane $H^{2}(K)$, and $d>0$ models a Lorentzian sphere, $S_{1}^{2}(K)$. By stereographic projections, $H^{2}(K)$ may be conveniently identified with the upper half-plane or disc models of $H^{2}(K)$.

We are mostly interested in space-like curves $\gamma(x) \subset R_{1}^{3}$; in this case, we may assume unit-speed parametrization, $\left\langle\gamma_{x}, \gamma_{x}\right\rangle=1$. We consider an orthogonal frame $\{T, N, i B\}$ along $\gamma$, satisfying $\langle T, T\rangle=\langle N, N\rangle=-\langle i B, i B\rangle=1$, and the following Frenet system:

$$
\gamma_{x}=T, T_{x}=\kappa N, N_{x}=-\kappa T+\tau i B, i B_{x}=\tau N .
$$

Note that the tangent indicatrix, $T(x)$, of $\gamma$ may be regarded as a curve in $S_{1}^{2}(1)$, while the binormal indicatrix, $i B(x)$, is a curve in $H^{2}(-1)$.

As our notation suggests, (FS) may be thought of, alternatively, as the classical Frenet equations for an orthonormal frame $\{T, N, B\}$, but with imaginary torsion $i \tau$ (and positive curvature $\kappa$ ). While this simple heuristic will suffice, for most purposes, it may be useful also to mention the Lie-algebraic background.

Given a semi-simple Lie group $G$ with Lie algebra $g$ and Cartan-Killing form $\langle$,$\rangle ,$ generalized Frenet equations for curves in $(g,\langle\rangle$,$) may be defined, via the adjoint$ representation, from differential equations on $G$. The choice $g=s u(2) \cong E^{3}$ yields (in one case) the classical Frenet equations, while $g=\operatorname{sl}(2, R) \cong R_{1}^{3}$ leads (in one case) to the above equations for space-like Lorentzian curves. The choice $g=\operatorname{sl}(2, C) \cong C^{3}$ provides a unified context, in which notions such as imaginary torsion may be used to relate the two types of curves. To illustrate, we now fix convenient notation for the Lorentzian case, first identifying $s l(2, R)$ with $R_{1}^{3}$ using the basis

$$
e_{0}=\frac{1}{2}\left(\begin{array}{cc}
1 & 0 \\
0 & -1
\end{array}\right), \quad e_{1}=\frac{1}{2}\left(\begin{array}{ll}
0 & 1 \\
1 & 0
\end{array}\right), \quad e_{2}=\frac{1}{2}\left(\begin{array}{cc}
0 & 1 \\
-1 & 0
\end{array}\right) .
$$

Then the commutator bracket $[A, B]=A B-B A$ on $s l(2, R)$ is given by $\left[e_{0}, e_{1}\right]=$ $e_{2},\left[e_{0}, e_{2}\right]=e_{1},\left[e_{1}, e_{2}\right]=-e_{0}$, and the Cartan-Killing form is the above metric, 
$\langle A, B\rangle=\frac{1}{2} \operatorname{tr}(A B)$. Let $\Phi=\left(\begin{array}{ll}a & b \\ c & d\end{array}\right)$, with $a d-b c=1$, and consider the $S L(2, R)$ Lie system:

$$
\Phi_{x}=Q \Phi=\frac{1}{2}\left(\begin{array}{cc}
-\tau & -\kappa \\
\kappa & \tau
\end{array}\right) \Phi .
$$

To obtain (FS), set $T=\gamma_{x}=\Phi^{-1} e_{0} \Phi, N=-\Phi^{-1} e_{1} \Phi, i B=-\Phi^{-1} e_{2} \Phi$, and apply bracket relations to the formula $\frac{d}{d x} \Phi^{-1} V \Phi=\Phi^{-1} V_{x} \Phi+\Phi^{-1}[V, Q] \Phi$; Ad-invariance of $\langle$,$\rangle gives the required properties of the frame \{T, N, i B\}$.

Now suppose $\gamma$ happens to have constant torsion $\tau$. In this case, we define the transform $\tilde{\gamma}$ of $\gamma$ by the formula:

$$
\tilde{\gamma}=\gamma+\frac{2 C}{C^{2}-\tau^{2}}(\cos \eta T+\sin \eta N)
$$

Here, $C=$ constant $\neq \pm \tau$, and $\eta=\eta(x)$ solves the differential equation

$$
\eta_{x}=C \sin \eta-\kappa, \quad \eta\left(x_{0}\right)=\eta_{0},
$$

which implies $w=\tan (\eta / 2)$ satisfies the Riccati equation,

$$
w_{x}=C w-\frac{\kappa}{2}\left(1+w^{2}\right), \quad w\left(x_{0}\right)=w_{0} .
$$

Thus, the new curve $\tilde{\gamma}$ depends on: a) the parameter $C$ which controls the constant distance from $p=\gamma(x)$ to $\tilde{p}=\tilde{\gamma}(x)$, and b) the initial condition $\eta_{0}$ (or $w_{0}$ ), whose variation corresponds to sweeping out a circle of possible points $\tilde{p}$ in the osculating plane of $\gamma$ at $p$.

Proposition 1. Let $\gamma \subset R_{1}^{3}$ be a unit speed curve of constant torsion $\tau$ and curvature $\kappa$. Then (BTG) and (ODE) define a new unit speed curve $\tilde{\gamma} \subset R_{1}^{3}$ of constant torsion $\tilde{\tau}=\tau$ and curvature $\tilde{\kappa}=\kappa-2 C \sin \eta$, depending on parameters $C$ and $\eta_{0}$.

Proof. By direct (but tedious) computation, one may verify that $\tilde{\gamma}$ has speed, curvature, and torsion as claimed with Frenet system:

$$
\begin{aligned}
\tilde{T} & =\tilde{\gamma}_{s}=\left(1-\delta C Y^{2}\right) T+\delta C X Y N+(\delta \tau Y) i B \\
& =T+(1-\cosh (\zeta)) Y(X N-Y T)-\sinh (\zeta) Y i B \\
\tilde{N} & =\delta C X Y T+\left(1-\delta C X^{2}\right) N-(\delta \tau X) i B \\
& =N-(1-\cosh (\zeta)) X(X N-Y T)+\sinh (\zeta) X i B \\
\widetilde{i B} & =\delta \tau Y T-\delta \tau X N+(1-\delta C) i B \\
& =\cosh (\zeta) i B+\sinh (\zeta)(X N-Y T) .
\end{aligned}
$$

Here, $X(x)=\cos \eta(x), Y(x)=\sin \eta(x)$, and $\delta=\frac{2 C}{C^{2}-\tau^{2}}=-\sinh (\zeta) / \tau\left(\tanh \left(\frac{\zeta}{2}\right)=\frac{C}{\tau}\right)$.

Remark 1. We refer to BTG as a Bäcklund transform for curves, even though it is really only half of a BT in the standard sense of soliton theory; the construction is of geometric interest, even in the absence of time evolution. In this context, it should be noted that the Riccati equation (Ric) plays a dual role. Recall, any Lie system on $S L(2, C)$ implies a Riccati equation. In particular, (LS) implies the following real Riccati equation for $y=a / c$ : $y_{x}=-\tau y-\frac{\kappa}{2}\left(1+y^{2}\right)$. So $y$ satisfies (Ric) for $C=-\tau$. (The Euclidean Frenet equations come from an $S U(2)$ Lie system, leading, e.g., to the complex Riccati equation $z_{x}=i \tau z-\frac{\kappa}{2}\left(1+z^{2}\right)$; one may recover (Ric) by replacing $\tau$ with $i \tau$.) 
Now suppose we have explicitly computed a constant torsion curve $\gamma(x)$, e.g., by solving (LS) for some $\kappa(x)$ and $\tau=$ const. By general theory of ODE's, the solution to (LS) depends analytically on the parameter $\tau$; so it is reasonable to suppose, further, we have solved (LS), for all (real) values of $\tau$ - by analytic continuation. Then, in effect, we have already solved (Ric) and can therefore write down all Bäcklund transforms $\tilde{\gamma}$ of $\gamma$ (in fact, of all constant torsion curves with curvature $\kappa(x))$.

Remark 2. The above formulas are closely related to Euclidean counterparts; in fact, replacing $\tau$ with $i \tau$ in the formulas of [C-I], we recover exactly the above expressions for $\tilde{\gamma}, \tilde{\kappa}, \tilde{T}, \tilde{N}, \widetilde{i B}$. The equation (Ric) is unaffected - in particular, real and the corresponding continuation argument in the Euclidean case is thus complex analytic continuation (see $[\mathrm{C}-\mathrm{I}])$. We note that the frame expressions in $[\mathrm{C}-\mathrm{I}]$ may be written: $\tilde{T}=\left(1-d C Y^{2}\right) T+d C X Y N+(d \tau Y) B=T+(1-\cos (\theta)) Y(X N-Y T)+$ $\sin (\theta) Y B, \tilde{N}=d C X Y T+\left(1-d C X^{2}\right) N-(d \tau X) B=N-(1-\cos (\theta)) X(X N-Y T)-$ $\sin (\theta) X B$, and $\tilde{B}=-d \tau Y T+d \tau X N+(1-d C) B=\cos (\theta) B+\sin (\theta)(X N-Y T)$, where $d=\frac{2 C}{C^{2}+\tau^{2}}$. (For comparison with the above, set $\zeta=i \theta$.) In the context of the classical Bäcklund transformation for pseudospherical surfaces, $\theta$ is the constant angle between 'old' and 'new' surface normals, $n=B$ and $\tilde{n}=\tilde{B}$, at corresponding points.

The hyperbolic plane version of BTG is obtained using the following simple fact: as in the Euclidean case, a constant torsion (unit speed) curve may be reconstructed from its binormal. Here we use the rescaled binormal indicatrix, $\beta= \pm i B / \tau$, a unit speed curve in $H^{2}\left(-\tau^{2}\right)$ with geodesic curvature $\kappa_{\beta}= \pm \kappa$, and Frenet frame $T_{\beta}= \pm N, N_{\beta}=\mp T$. For $C \neq \pm \tau$, we define the transform $\tilde{\beta}= \pm \widetilde{i B} / \tau$ of $\beta$ :

$$
\tilde{\beta}=\frac{C^{2}+\tau^{2}}{\tau^{2}-C^{2}} \beta+\frac{2 C}{\tau^{2}-C^{2}}\left(\cos \eta T_{\beta}+\sin \eta N_{\beta}\right) .
$$

We note that $\beta$ and $\tilde{\beta}$ lie in opposite sheets of the hyperboloid $u^{2}+v^{2}-w^{2}=-1 / \tau^{2}$ when $\tau^{2}-C^{2}<0$. Identifying the two copies, $\pm H^{2}\left(-\tau^{2}\right)$, the above proposition thus translates to:

Proposition 2. Let $\beta \subset H^{2}\left(-\tau^{2}\right) \subset R_{1}^{3}$ be a unit speed curve with geodesic curvature $\kappa$. Then (BTB) and (ODE) define a new unit speed curve $\tilde{\beta} \subset H^{2}\left(-\tau^{2}\right)$ with geodesic curvature $\tilde{\kappa}=\kappa-2 C \sin \eta$, depending on parameters $C$ and $\eta_{0}$.

Remark 3. Ivey has recently considered a Bäcklund transformation for pseudospherical surfaces and constant torsion curves in the context of 3-dimensional, Riemannian space forms [Iv]. One easily checks that our formula (BTB) is the zero torsion case (not to be confused with setting our $\tau$ equal to zero!) of Ivey's formula for $H^{3}$. Perhaps it would be natural to approach (BTG) via the Lorentzian analogue of pseudospherical surfaces, but this is not directly relevant to our present purposes. Incidentally, the analytic continuation argument mentioned earlier has a nice geometric interpretation in the context of (BTB). Regard LS as 'Frenet equations' for a curve $\beta \subset H^{2}\left(-\tau^{2}\right)$ of geodesic curvature $\kappa(x)$. Solving LS for another parameter value, $\hat{\tau}$, corresponds to finding a curve $\hat{\beta} \subset H^{2}\left(-\hat{\tau}^{2}\right)$ with the same curvature function. Now the transformation $\beta \rightarrow \hat{\beta}$ may be thought of as rolling the surface $H^{2}\left(-\tau^{2}\right) \subset R_{1}^{3}$ on the surface $H^{2}\left(-\hat{\tau}^{2}\right) \subset R_{1}^{3}$, along the curve $\beta$, as the contact point traces out $\hat{\beta}$ on $H^{2}\left(-\hat{\tau}^{2}\right)$. 
We now introduce time evolution, via the modified Korteweg-de Vries equation, regarded as an equation for curvature:

$(\mathrm{mKdV})$

$$
\kappa_{t}=\kappa_{x x x}+\frac{3}{2} \kappa^{2} \kappa_{x}
$$

For arguments in the next section, we briefly recall the derivation of $\mathrm{mKdV}$ as compatibility condition for an overdetermined linear system with potential $q=-\kappa$ and spectral parameter $\lambda$ :

$$
\begin{gathered}
\Psi_{x}=L(x, t ; \lambda) \Psi=\frac{1}{2}\left(\begin{array}{cc}
\lambda & q(x, t) \\
-q(x, t) & -\lambda
\end{array}\right) \Psi, \\
\Psi_{t}=M(x, t ; \lambda) \Psi=\frac{1}{2}\left(\begin{array}{cc}
A(x, t ; \lambda) & B(x, t ; \lambda) \\
C(x, t ; \lambda) & -A(x, t ; \lambda)
\end{array}\right) \Psi .
\end{gathered}
$$

Defining covariant derivative operators $D_{x}=\frac{\partial}{\partial x}-L(x, t ; \lambda)$ and $D_{t}=\frac{\partial}{\partial t}-$ $M(x, t ; \lambda)$, one may express the condition $\Psi_{x t}=\Psi_{t x}$ as $\left[D_{x}, D_{t}\right]=0$ (the zero curvature condition (ZCC)). Invoking the appropriate polynomial ansatz,

$$
A=\sum_{k=0}^{n} \lambda^{2 k+1} A_{2 k+1}, B+C=2 \sum_{k=0}^{n} \lambda^{2 k+1} S_{2 k+1}, B-C=2 \sum_{k=0}^{n} \lambda^{2 k} T_{2 k},
$$

one determines $A, B, C$ by substituting into (ZC) and equating coefficients of all powers of $\lambda$ to zero. The $\lambda^{0}$ term gives the $n$th equation in the $\mathrm{mKdV}$ hierarchy, $q_{t}=\frac{\partial}{\partial x} T_{0}$, a PDE for potential $q(x, t)$ evolving isospectrally (no $\lambda$-dependence).

In particular, $n=1$ gives

(ABC) $\quad A=\frac{q^{2}}{2} \lambda+\lambda^{3}, B=q_{x x}+\frac{q^{3}}{2}+q_{x} \lambda+q \lambda^{2}, C=-q_{x x}-\frac{q^{3}}{2}+q_{x} \lambda-q \lambda^{2}$

and subsequently $q_{t}=q_{x x x}+\frac{3}{2} q^{2} q_{x}$, the mKdV equation.

More generally, one may derive recursive formulas for the higher order commuting flows in the mKdV hierarchy, as well as the corresponding Hamiltonians $H_{n}$ for these equations. We omit these formulas - we will not use them - but list the first few Hamiltonians, and note that it is a straightforward exercise to check that they are conserved by mKdV:

$$
H_{0}=\int q d x, H_{1}=\int \frac{q^{2}}{2} d x, H_{2}=\int \frac{\left(q_{x}\right)^{2}}{2}-\frac{q^{4}}{8} d x .
$$

We recall one more fact about mKdV: a Bäcklund transformation, giving a 'new' solution, $\tilde{q}=2 \tilde{u}_{x}$, from an 'old', $q=2 u_{x}$, may be described by the following pair of equations involving the average angle $-\eta=u+\tilde{u}=\int \frac{q+\tilde{q}}{2} d x$ and modulation angle $\theta=u-\tilde{u}=\int \frac{q-\tilde{q}}{2} d x$ :

$$
\begin{gathered}
\theta_{x}=C \sin \eta \\
\theta_{t}=C\left[\frac{1}{2} q^{2} \sin \eta-q_{x} \cos \eta-C \eta_{x}\right] .
\end{gathered}
$$

For these equations we refer the reader to Theorem 5.4.13 of [Ro] ( $\operatorname{set} q=2 v$, $\alpha=1, \beta=C$, and replace $t$ by $-t$ ).

Returning now to curves, the following proposition shows how $\mathrm{mKdV}$ arises naturally from geometric evolution equations: 
Proposition 3. a) For $\gamma$ in $E^{3} \cong s u(2)$ or $R_{1}^{3} \cong s l(2, R)$, the evolution equation

$$
\gamma_{t}=\gamma_{x x x}-3 \tau\left[\gamma_{x}, \gamma_{x x}\right]+\left(\frac{3}{2}\left\|\gamma_{x x}\right\|^{2}-3 \tau^{2}\right) \gamma_{x}
$$

preserves unit speed $\left\|\gamma_{s}\right\|=\left\|\gamma_{x}\right\|=1$ and constant torsion $\tau$, while inducing evolution of curvature $\kappa$ by $m K d V$.

b) For $\beta$ a curve in $E^{2}, H^{2}$, or $S^{2}$, the evolution equation

$$
\beta_{t}=\nabla_{\beta_{x}}^{2} \beta_{x}+\left(\frac{3}{2}\left\|\nabla_{\beta_{x}} \beta_{x}\right\|^{2}-G\right) \beta_{x}=\left(\frac{\kappa^{2}}{2}-G\right) T+\kappa_{x} N
$$

preserves unit speed $\left\|\beta_{x}\right\|=1$, while $\kappa$ satisfies $m K d V$. Here, $G$ is the scalar curvature of $E^{2}, H^{2}$, or $S^{2}$.

Proof. The $E^{3}$ case of a) was introduced in [La], and the $E^{2}$ case of b) was discussed in [G-P]. More general results were given in [L-P 2, L-P 3] (including higher dimensional analogues of $\mathrm{b}$ )). The hyperbolic case of b) is not discussed in [L-P 2, L-P 3], but one can easily apply variation formulas from [L-S 1]. Similarly, the Lorentzian case of a) is not treated in the above references, but the necessary curvature and torsion variation formulas are similar to those in [L-P 1].

To tie everything together, we bring the curve transformations BTG (BTB) back into the picture. Note that ODE may be regarded as the $x$-half of the above Bäcklund transformation for $\mathrm{mKdV}$. That is, BTx describes precisely the same relationship between $\kappa=-q$ and $\tilde{\kappa}=-\tilde{q}$ as does ODE (with $\tilde{\kappa}=\kappa-2 C \sin \eta$ ). Of course, BTG (BTB) does not fully relate two curve dynamics, since the initial condition $\eta_{0}$ in ODE depends on time; however, given a curve $\gamma(s, t)(\beta(s, t))$ evolving by CTP (CE), we can solve BTx, BTt for $\eta(s, t)$, then apply BTG (BTB).

Example 1. From a geometry/graphics standpoint, the case of constant curvature $\kappa(x, t)=\kappa=-q$ is already interesting. To save space, we neglect constant torsion curves, and discuss only periodic $\beta, \tilde{\beta} \subset H^{2}(-1)$ (relevant to the tori of revolution in Section 3). Thus, we take $\beta$ to be a circle, i.e., a curve with geodesic curvature $\kappa^{2}>1 ; \beta$ is one of the closed curves obtained by intersecting the hyperboloid $-1=\langle V, V\rangle=u^{2}+v^{2}-w^{2}$ with a plane of 'slope' less than one. Other planes cut out horocycles $(\kappa=1)$, parallel curves $(0<\kappa<1)$, and geodesics $(\kappa=0)$, none of which are closed. For convenience, we use the horizontal (sliding) circle solution $\beta(x, t)=(g \cos \delta, g \sin \delta, f), f=\cosh \nu, g=\sinh \nu, \delta=\left(x+\left(\frac{\kappa^{2}}{2}+1\right) t\right) / g$ which has arclength parametrization, curvature $\kappa=\operatorname{coth} \nu$, and Frenet frame $T_{\beta}=$ $(-\sin \delta, \cos \delta, 0), N_{\beta}=(-f \cos \delta,-f \sin \delta,-g)$.

To get a periodic (rather than decaying) curvature function $\tilde{\kappa}$, we take $C^{2}<\kappa^{2}$ in (ODE), and obtain the elementary solution:

$$
\eta=2 \arctan \left[\frac{1}{\kappa}(C-\rho \tan \omega)\right], \omega=\frac{\rho x}{2}+\mu, \rho=\sqrt{\kappa^{2}-C^{2}}
$$


where the time-dependence of the constant of integration $\mu=\mu(t)$ is easily determined in this case. Writing $X=\cos \eta, Y=\sin \eta, \eta_{x}=C Y-\kappa$ (ODE), $X_{x}=-Y(C Y-\kappa), Y_{x}=X(C Y-\kappa)$, and $\tilde{\kappa}=\kappa-2 C Y$, one straightforwardly computes $\tilde{\kappa}_{x x x}+\frac{3}{2} \tilde{\kappa}^{2} \tilde{\kappa}_{x}=-C\left(\kappa^{2}+2 C^{2}\right) Y_{x}$ and $\tilde{\kappa}_{t}=-\frac{4 C}{\rho} \mu_{t} Y_{x}$. Thus, we may take $\omega=\frac{\rho x}{2}+\frac{\rho}{4}\left(\kappa^{2}+2 C^{2}\right) t$, and $\tilde{\kappa}$ is a travelling wave solution of $\mathrm{mKdV}$. The corresponding curve $\tilde{\beta}$, evolving by (CE), is simply rotating (and 'slipping' along itself).

More explicitly, applying (BTB) (with $\tau=-1$ ), we compute the coordinates of $\tilde{\beta}=(u, v, w)$ :

$$
\begin{aligned}
u & =R g \cos \delta+S \sin \delta X+S f \cos \delta Y, \\
v & =R g \sin \delta-S \cos \delta X+S f \sin \delta Y, \\
w & =R f+S g Y, \quad R=\frac{C^{2}+1}{1-C^{2}}, \quad S=-\frac{2 C}{1-C^{2}}, \\
X & =\frac{\rho^{2} \cos 2 \omega+\rho C \sin 2 \omega}{\kappa^{2}+C^{2} \cos 2 \omega-\rho C \sin 2 \omega}, \quad Y=\frac{\kappa C+\kappa C \cos 2 \omega-\kappa \rho \sin 2 \omega}{\kappa^{2}+C^{2} \cos 2 \omega-\rho C \sin 2 \omega} .
\end{aligned}
$$

Evidently, the closure ( $x$-periodicity) condition for $\tilde{\beta}$ is simply rationality of $g \rho$. Thus, one may easily generate closed examples $\tilde{\beta}$ by computer with discrete rotational symmetries. Figure 1a) shows a five-fold example (with $g=1, f=\kappa=$ $\sqrt{2}, C=-\sqrt{7} / 4, \rho=5 / 4, R=23 / 9, S=8 \sqrt{7} / 9$, and period $P=8 \pi)$, along with its isometric (stereographic) image in the Poincare disc (with projection point $(0,0,-1), \Pi(u, v, w)=z=(u+i v) /(1+w))$. Figure 1b) shows the isometric image, $\sigma$, in the half-plane model (under $z \mapsto Z=U+i V=(z+1) /(1-z)=$ $(1+i v) /(w-u))$. Figure 2 depicts (CE) for $\sigma$ over a congruence period. Of course, the elementary hyperbolic symmetry of the curve and its rotational motion are much more transparent in Figure 1a); but as we'll explain in the next section, Figure 2 is to be viewed with Euclidean eyes!

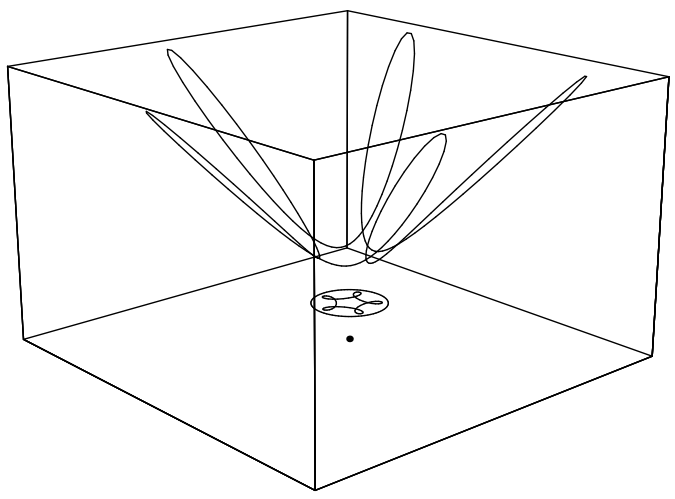

(a)

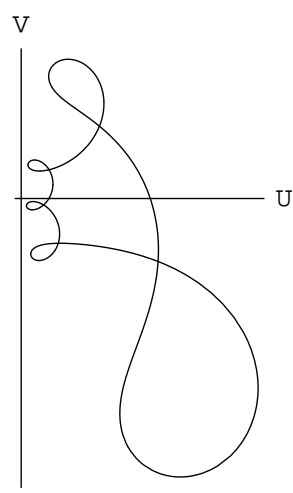

(b)

FiguRE 1. a) Initial curve $\tilde{\beta}$ in hyperboloid model of $H^{2}$ and stereographic image in Poincare disc. b) Initial curve in half-plane model. 

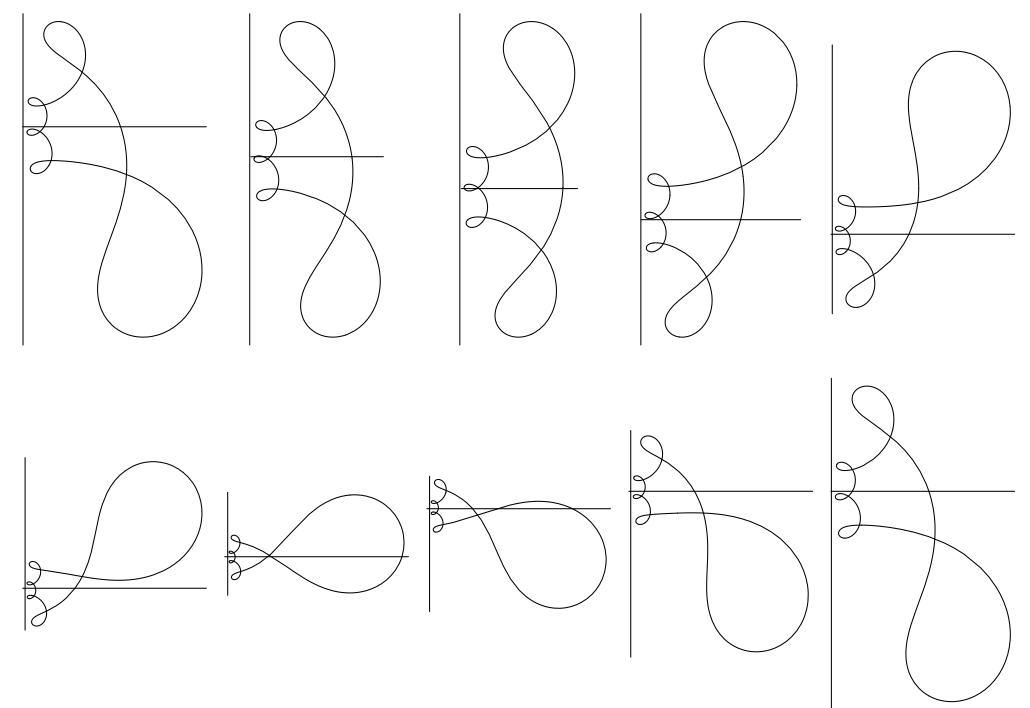

Figure 2. Evolution of $\sigma$ in half-plane $H^{2}$.

\section{TAImanov's SURface EVOlution}

In recent years, a number of authors have discussed generalized Weierstrass representations (WR) of surfaces, and connections to integrable systems theory. In particular, Taimanov [Ta 1] has shown how the modified Novikov-Veselov equation

$$
U_{t}=\left(U_{z z z}+3 U_{z} V+\frac{3}{2} U V_{z}\right)+\left(U_{\bar{z} \bar{z} \bar{z}}+3 U_{\bar{z}} \bar{V}+\frac{3}{2} U \bar{V}_{\bar{z}}\right), V_{\bar{z}}=\left(U^{2}\right)_{z}
$$

may be used to induce (via WR) geometrically interesting evolutions of surfaces in $E^{3}$. We review the basic constructions, focussing on the surface of revolution case, as in [Ta 2].

Fixing $t$ for the moment, let $\psi(x, y)=\left(\begin{array}{c}\psi_{1} \\ \psi_{2}\end{array}\right)$ be a zero eigenfunction of the Dirac Operator, $D=\left(\begin{array}{cc}\partial_{z} & -U \\ U & \partial_{\bar{z}}\end{array}\right)$. Then it turns out, for $U$ real, the following WR formulas give a well-defined parametrized surface $\Sigma=\left(Z_{1}, Z_{2}, Z_{3}\right)$ :

$$
\begin{aligned}
Z_{1} & =\frac{i}{2} \int\left(\bar{\psi}_{1}^{2}+\psi_{2}^{2}\right) d z-\left(\bar{\psi}_{2}^{2}+\psi_{1}^{2}\right) d \bar{z} \\
Z_{2} & =\frac{1}{2} \int\left(\bar{\psi}_{1}^{2}-\psi_{2}^{2}\right) d z-\left(\bar{\psi}_{2}^{2}-\psi_{1}^{2}\right) d \bar{z} \\
Z_{3} & =-\int \bar{\psi}_{1} \psi_{2} d z+\psi_{1} \bar{\psi}_{2} d \bar{z}
\end{aligned}
$$

The representation is particularly simple for surfaces of revolution; here we assume no $y$-dependence in the potential $(U=U(x))$, and $\psi$ has the form $\psi(x, y)=$ 
$e^{i y / 2}\left(\begin{array}{c}r(x) \\ s(x)\end{array}\right)$. In this case, the zero eigenfunction may be taken to satisfy

$$
\frac{d}{d x} \psi=\frac{d}{d x}\left(\begin{array}{c}
r \\
s
\end{array}\right)=\frac{1}{2}\left(\begin{array}{ll}
-1 & q \\
-q & 1
\end{array}\right)\left(\begin{array}{l}
r \\
s
\end{array}\right)
$$

where $q(x, t)=4 U$. The profile curve of $\Sigma(x, y)=\left(\theta(x) \cos \frac{y}{2}, \theta(x) \sin \frac{y}{2}, \phi(x)\right)$ may be written

$$
\sigma(x)=(\theta(x), 0, \phi(x)), \theta_{x}=s^{2}-r^{2}, \phi_{x}=2 r s .
$$

Writing MS as $r_{x}=\frac{1}{2}(-r+s q), s_{x}=\frac{1}{2}(s-r q)$, one finds that $\left(s^{2}+r^{2}\right)_{x}=$ $s^{2}-r^{2}=\theta_{x}, \theta_{x x}=\left(s^{2}-r^{2}\right)_{x}=s^{2}+r^{2}-\phi_{x} q, \phi_{x x}=\left(s^{2}-r^{2}\right) q=\theta_{x} q$. We obtain $\theta$ from the first of these (essentially normalizing the antiderivative), and collect a number of useful identities:

$$
\begin{gathered}
\theta=s^{2}+r^{2}, \quad \theta^{2}=\theta_{x}^{2}+\phi_{x}^{2}, \quad \theta_{x x}=\theta-\phi_{x} q, \\
\phi_{x x}=\theta_{x} q, \quad r^{2}=\frac{1}{2}\left(\theta-\theta_{x}\right), \quad s^{2}=\frac{1}{2}\left(\theta+\theta_{x}\right) .
\end{gathered}
$$

In particular, it follows that the first fundamental form of $\Sigma$ is given by $d s^{2}=$ $\theta^{2}\left(d x^{2}+d y^{2}\right) ; \theta(x)$ is, up to scaling, the unique function consistent with $\Sigma=$ $(\theta(x) \cos y, \theta(x) \sin y, \phi(x))$ defining a conformal parametrization of $\Sigma$. Conversely, given a function $\theta(x)$ (with $\left(\phi_{x}\right)^{2}=: \theta^{2}-\left(\theta_{x}\right)^{2}>0$ ), one obtains a conformally parametrized surface of revolution $\Sigma$ using the above formulas. $\Sigma$ will be a closed surface provided $\theta(x)$ is periodic and $\phi_{x}$ integrates to zero over a period of $\theta(x)$; for $\theta>0$, one obtains an immersed torus of revolution. (The assumption $\theta(x)>0$ will be used, as needed, to simplify the discussion - otherwise, one might still get an immersed sphere, with most of our conclusions remaining valid.)

To introduce time-dependence for $\Sigma$, note that when $\lambda=-1$, (LSx) is exactly (MS). This suggests the natural connection between WR and integrable systems. Specifically, consider $\Psi(x, t ; \lambda)$ satisfying (LSx), (LSt) with (ABC) - consequently $q(x, t)$ satisfies $\mathrm{mKdV}$. Set $\lambda=-1$, and then define an evolving curve $\sigma(x, t)$ by (PC), and corresponding surface of revolution $\Sigma(x, y, t)$. Henceforth, we will refer to this evolution of conformally parametrized surfaces as the Taimanov evolution (TE). Similarly, higher order surface evolutions (TEn) may be defined using the mKdV hierarchy.

Next we describe the connection between surfaces of revolution $\Sigma \subset E^{3}$ and curves in the hyperbolic plane (see [L-S 2]). Consider the following metric on $M^{3}=$ $E^{3} \backslash\left\{Z_{2}\right.$ axis $\}$, evidently pointwise conformal to the Euclidean metric: $\langle\langle V, W\rangle\rangle=$ $\frac{V \cdot W}{Z_{1}^{2}}$, where $V \cdot W$ denotes the usual dot product on $E^{3}$. Note that the correspondence $(\theta, 0, \phi) \leftrightarrow w=\phi+i \theta$ identifies the half-plane $\left\{\left(Z_{1}, Z_{2}, Z_{3}\right): Z_{1}>0, Z_{2}=0\right\} \subset$ $M^{3}$ with the upper half-plane model of the hyperbolic plane $H^{2}=H^{2}(-1)$, and $M^{3}$ itself with the Riemannian product $M^{3}=H^{2} \times S^{1}$. 
Proposition 4. Let $\Sigma \subset E^{3}$ be a surface of revolution with profile curve $\sigma(x)=$ $(\theta(x), 0, \phi(x)) \subset H^{2}(-1)$, as above. Then

a) $\sigma(x)$ has unit speed, and geodesic curvature $\kappa=-q+\frac{2 \phi_{x}}{\theta}$, and

b) for $\sigma$ closed, $\Sigma$ has (rectangular) conformal type $\mathcal{C}(\Sigma)=\operatorname{Length}(\sigma) / 2 \pi$ and Willmore functional $\mathcal{W}(\Sigma)=\int_{\Sigma} H^{2} d A=\frac{\pi}{2} \int_{\sigma} \kappa_{g}^{2} d s$.

Proof. The first part of a) is self-evident. To prove the second part of a), we express the (positively oriented) Frenet frame along $\sigma$ in terms of coordinate vector fields: $T=\phi_{x} \partial_{\phi}+\theta_{x} \partial_{\theta}, N=-\theta_{x} \partial_{\phi}+\phi_{x} \partial_{\theta}$. From basic covariant derivative expressions $\nabla_{\partial_{\phi}} \partial_{\phi}=\frac{1}{\theta} \partial_{\theta}, \nabla_{\partial_{\phi}} \partial_{\theta}=\nabla_{\partial_{\theta}} \partial_{\phi}=-\frac{1}{\theta} \partial_{\phi}, \nabla_{\partial_{\theta}} \partial_{\theta}=-\frac{1}{\theta} \partial_{\theta}$, follows $\nabla_{T} T=$ $\frac{1}{\theta}\left[\left(\theta \phi_{x x}-2 \phi_{x} \theta_{x}\right) \partial_{\phi}+\left(\theta \theta_{x x}+\phi_{x}^{2}-\theta_{x}^{2}\right) \partial_{\theta}\right]$. Using $\phi_{x x}=\theta_{x} q$ and $\theta \theta_{x x}-\theta_{x}^{2}=$ $-\theta q \phi_{x}+\phi_{x}^{2}$, we rewrite the above as $\nabla_{T} T=\frac{1}{\theta}\left[\left(\theta \theta_{x} q-2 \phi_{x} \theta_{x}\right) \partial_{\phi}+\left(2 \phi_{x}^{2}-\theta q \phi_{x}\right) \partial_{\theta}\right]=$ $\frac{2 \phi_{x}-\theta q}{\theta} N=-\left(q-\frac{2 \phi_{x}}{\theta}\right) N=\kappa_{g} N$.

For the first part of $b$ ), we recall that any torus may be represented as a parallelogram, and tori of revolution are rectangular. In the metric on $\Sigma$ induced by $\langle\langle\rangle$,$\rangle , the edge lengths of \Sigma$ are $2 \pi$ and Length $(\sigma)$.

The second part b) follows from the well-known conformal invariance property of $\int_{\Sigma} H^{2}+\bar{K} d A$ (see, e.g., [Ch] or [We]). Here, $H$ denotes mean curvature of $\Sigma, \bar{K}$ is the ambient sectional curvature along $\Sigma$, which vanishes identically (for $\Sigma \subset E^{3}$, as well as for $\Sigma \subset M^{3}$ ), and $d A$ is the induced area element. (The formula $\mathcal{W}(\Sigma)=\frac{\pi}{2} \int_{\sigma} \kappa_{g}^{2} d s$ was used in [L-S 2] to prove the Willmore conjecture for surfaces of revolution, and to relate Willmore surfaces to elastic curves in $H^{2}$.)

Theorem 1. Let $\Sigma(x, y, t) \subset E^{3}$ be a surface of revolution with profile curve $\sigma(x, t)=(\theta(x, t), 0, \phi(x)) \subset H^{2}(-1)$, as above, evolving by the Taimanov evolution. Then $\sigma$ satisfies the unit speed-preserving curve evolution equation

$$
\sigma_{t}=\nabla_{\sigma_{x}}^{2} \sigma_{x}+\left(\frac{3}{2}\left\|\nabla_{\sigma_{x}} \sigma_{x}\right\|^{2}+1\right) \sigma_{x}=\left(\frac{\kappa^{2}}{2}+1\right) T+\kappa_{x} N
$$

The geodesic curvature $\kappa$ of $\sigma$ satisfies the modified Korteweg-de Vries equation, and is a Bäcklund transform of the potential $q$ of $\Sigma$.

The following geometric properties of $\sigma$ are preserved in time: closure, length, total curvature $\mathcal{H}_{0}$, total squared curvature $\mathcal{H}_{1}, \mathcal{H}_{2}$, etc. Consequently, the Taimanov evolution of $\Sigma$ preserves closure, conformal type, the Willmore functional $\mathcal{W}$, etc.

Proof. Introduce variables $w$ and $\eta$ according to $w=r / s=\tan (\eta / 2)$ and note:

a) $w$ satisfies the Riccati equation $w_{x}=-w-\frac{\kappa}{2}\left(1+w^{2}\right)$,

b) $\eta$ satisfies $\eta_{x}=q-\sin \eta=q-Y$ (i.e., ODE with $C=-1$ ),

c) (PC) may be rewritten as $\theta_{x}=\theta \cos \eta=\theta X, \phi_{x}=\theta \sin \eta=\theta Y$,

d) the geodesic curvature of $\sigma$ is given by $\kappa=-q+2 Y$.

[Observations b), d) suggest viewing $\kappa$ as a Bäcklund transform of $-q$, and $\sigma$ as a (half-plane representative of a) BT, $\tilde{\beta}$, of a curve $\beta \subset H^{2}$ with curvature $-q$. But $C=-1$ is precisely the excluded value in (BTB)! ]

We verify $(\mathrm{CE})$ by direct computation. To compute the right-hand-side, $W=$ $\left(\frac{\kappa^{2}}{2}+1\right) T+\kappa_{x} N$, we use $\kappa_{x}=-q_{x}+2 \eta_{x} X$ and $\frac{\kappa^{2}}{2}=\frac{q^{2}}{2}-2 \eta_{x} Y$ : 


$$
\begin{aligned}
W= & {\left[\phi_{x}\left(\frac{\kappa^{2}}{2}+1\right)-\theta_{x} \kappa_{x}\right] \partial_{\phi}+\left[\theta_{x}\left(\frac{\kappa^{2}}{2}+1\right)+\phi_{x} \kappa_{x}\right] \partial_{\theta} } \\
= & {\left[\phi_{x}\left(\frac{q^{2}}{2}-2 \eta_{x} Y+1\right)-\theta_{x}\left(-q_{x}+2 \eta_{x} X\right)\right] \partial_{\phi} } \\
& +\left[\theta_{x}\left(\frac{q^{2}}{2}-2 \eta_{x} Y+1\right)+\phi_{x}\left(-q_{x}+2 \eta_{x} X\right)\right] \partial_{\theta} \\
= & {\left[\theta_{x} q_{x}+\phi_{x}\left(\frac{q^{2}}{2}+1\right)-2 \eta_{x}\left(\phi_{x} Y+\theta_{x} X\right)\right] \partial_{\phi} } \\
& +\left[-\phi_{x} q_{x}+\theta_{x}\left(\frac{q^{2}}{2}+1\right)+2 \eta_{x}\left(\phi_{x} X-\theta_{x} Y\right)\right] \partial_{\theta} \\
= & {\left[\theta_{x} q_{x}+\phi_{x}\left(\frac{q^{2}}{2}+1\right)-2 \eta_{x} \theta\right] \partial_{\phi}+\left[-\phi_{x} q_{x}+\theta_{x}\left(\frac{q^{2}}{2}+1\right)\right] \partial_{\theta} . }
\end{aligned}
$$

For the left-hand-side of (CE), we write $\sigma_{t}=\phi_{t} \partial_{\phi}+\theta_{t} \partial_{\theta}$, and use (LSt), (ABC), etc., with $\lambda=-1$, to compute $\theta_{t}=2\left(r r_{t}+s s_{t}\right)=A\left(r^{2}-s^{2}\right)+(B+C) r s=$ $-\left(\frac{q^{2}}{2}+1\right)\left(r^{2}-s^{2}\right)-2 r s q_{x}=-\phi_{x} q_{x}+\theta_{x}\left(\frac{q^{2}}{2}+1\right)$. The computation of $\phi_{t}$ requires antidifferentiation of $\phi_{x t}=(2 r s)_{t}=2\left(r s_{t}+s r_{t}\right)=B s^{2}+C r^{2}=\frac{1}{2}(B+C) \theta+$ $\frac{1}{2}(B-C) \theta_{x}=-\theta q_{x}+\theta_{x}\left(q_{x x}+\frac{1}{2} q^{3}+q\right)$. Using the above formulas for $\theta_{x x}$ and $\phi_{x x}$, one verifies that $\theta_{x} q_{x x}=\left[\theta_{x} q_{x}-\theta q+\phi_{x}\left(\frac{q^{2}}{2}+1\right)\right]_{x}-\frac{1}{2} \theta_{x} q^{3}$, and subsequently $\phi_{x t}=\left[\theta_{x} q_{x}-2 \theta q+\phi_{x}\left(\frac{q^{2}}{2}+3\right)\right]_{x}$. Since $\eta_{x} \theta=(q-Y) \theta=\theta q-\phi_{x}$, the first component of $W$ is seen to agree with the expression for $\phi_{t}$ just obtained. (We are effectively normalizing an unimportant constant of integration, $\phi\left(x_{0}, t\right)$, which corresponds to isometry in $H^{2}$.) Thus we have verified (CE), and the rest of the theorem now follows from earlier discussion.

Remark 4. As noted in the proof, we cannot use (BTB) directly to relate two curves, $\beta$ and $\sigma \subset H^{2}$, with respective curvatures $-q$ and $\kappa$; though the Bäcklund transformation is perfectly well-behaved on the curvature level, (BTB) would place $\tilde{\beta}$ at infinity ( $\sigma$ on the boundary of the half-plane). One possibility is to use (BTB) to define a perturbation $\sigma_{C}$; one may then allow $C$ to approach the excluded value, $C=-1$, simultaneously applying translational isometries to keep $\sigma_{C}$ from wandering off to infinity in the limit. Such a renormalization could be made consistent with $\sigma(x, t)=\sigma_{-1}(x, t)$ satisfying (CE) exactly (but we have not found a natural description of this process).

On the other hand, one may directly apply (BTB) and Bäcklund transformation techniques to generate solutions to (TE). By the theorem, one is simply looking for solutions, $\sigma(x, t)$, to $(\mathrm{CE})$; for this, one is free to choose any convenient values for $C$. For instance, we may generate tori of revolution using the curves shown in Figure 2; Figures 3 and 4 show (one-half of) the surfaces $\Sigma$ generated by the first and sixth of these curves (respectively).

We are indebted to Ron Perline for producing the high-quality images in Figures 3 and 4 (using IRIS Explorer and software developed in Department of Mathematics and Computer Science, Drexel University). 


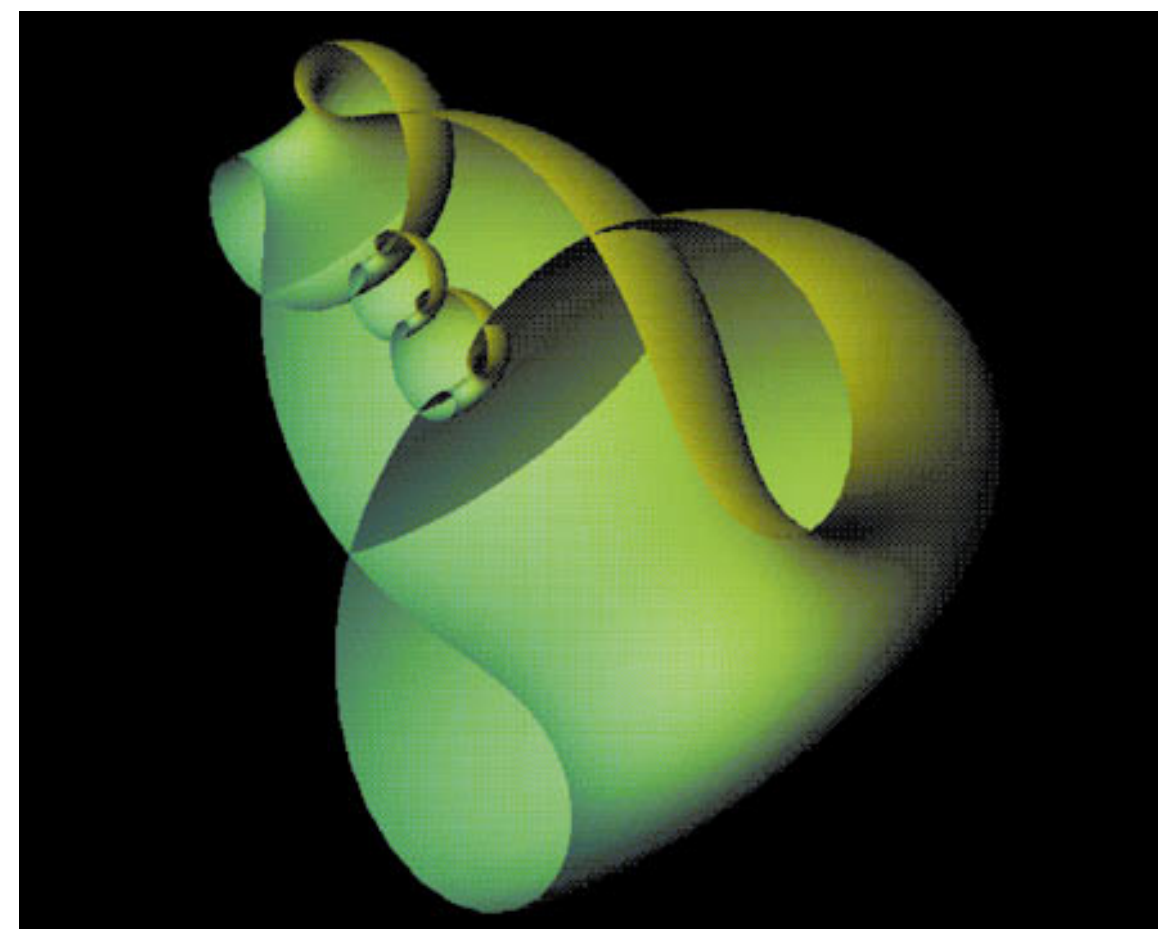

Figure 3. Surface of revolution from curve in $H^{2}$.

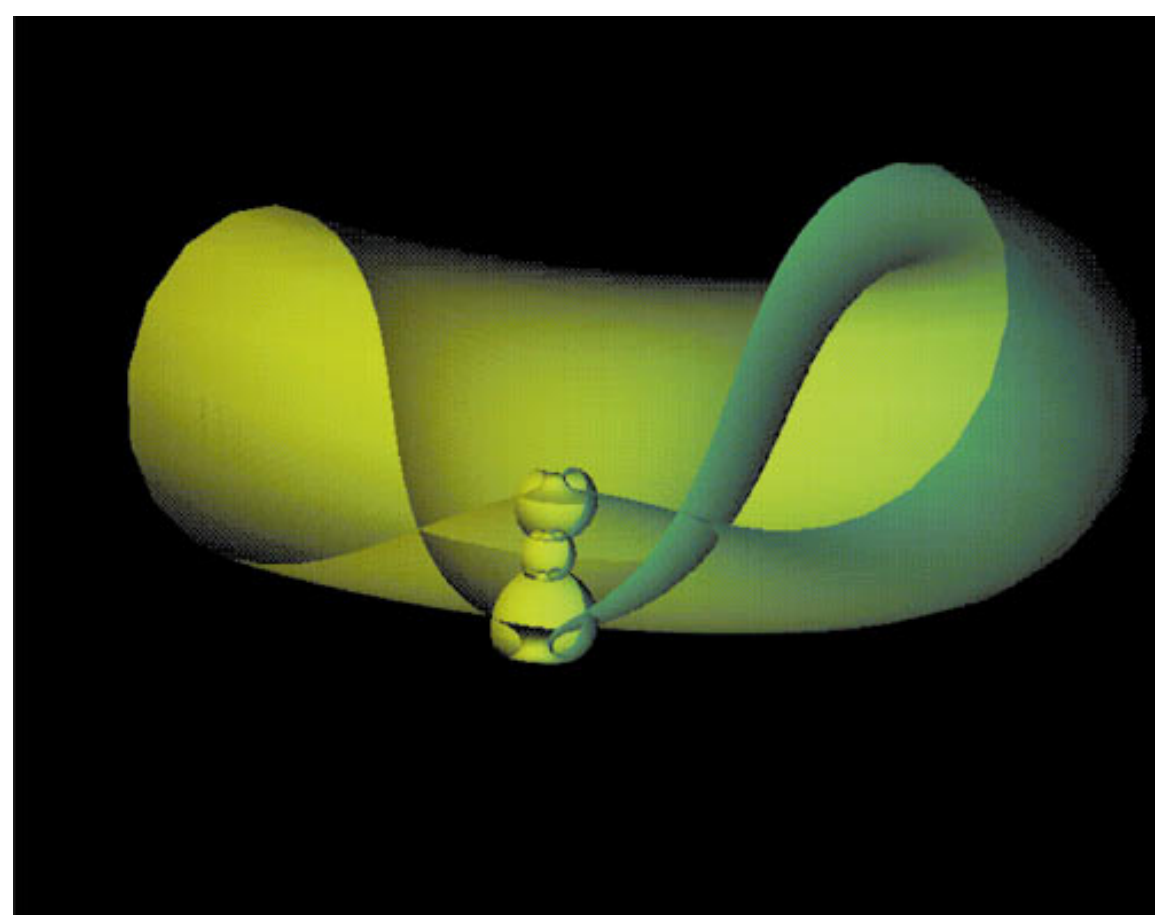

Figure 4. Surface of revolution at later time. 


\section{REFERENCES}

[C-I] A. Calini and T. Ivey, Bäcklund transformations and knots of constant torsion, J. Knot Theory and its Ramifications 7 (1998), p. 719. CMP 99:01

[Ch] B. Y. Chen, Some conformal invariants of submanifolds and their applications, Bol. Un. Mat. Ital. (4) 10 (1974). MR 51:6663

[G-P] R. Goldstein and D. Petrich, The Korteweg-de Vries hierarchy as dynamics of closed curves in the plane, Phys. Rev. Lett. 67 (1991), p. 3203. MR 92g:58050

[Iv] T. Ivey, Helices, Hasimoto surfaces and Bäcklund transformations, Preprint (1998).

[Ko] B. Konopelchenko, Induced surfaces and their integrable dynamics, Studies in Appl. Math. 96 (1996), p. 9. MR 96i:53011

[La] G. Lamb, Solitons and the motion of helical curves, 37 (1976), p. 235. MR 57:13250

[L-P 1] J. Langer and R. Perline, Poisson geometry of the filament equation, J. Nonlinear Sci. 1 (1991), p. 71. MR 92k:58118

[L-P 2] J. Langer and R. Perline, Local geometric invariants of integrable evolution equations, $J$. Math. Phys. 35 (1994), p. 1732. MR 95c:58095

[L-P 3] J. Langer and R. Perline, Curve motion inducing modified Korteweg-de Vries systems, Phys. Lett. A 239 (1998), pp. 36-40. CMP 98:10

[L-S 1] J. Langer and D. Singer, The total squared curvature of closed curves, J. Diff. Geom. 20 (1984), pp. 1-22. MR 86i:58030

[L-S 2] J. Langer and D. Singer, Curves in the hyperbolic plane and mean curvature of tori in 3-space, Bull. London Math Soc. 16 (1984), pp. 531-534. MR 85k:53006

[Ro] C. Rogers, Bäcklund transformations in soliton theory, in Soliton theory: a survey of results, ed. A. P. Fordy, St. Martin's Press, 1990. CMP 91:07

[Ta 1] I. Taimanov, Modified Novikov-Veselov equation and differential geometry of surfaces, Preprint November 1995 (da-ga 9511005), Translations Amer. Math. Soc., Ser. 2, 179, 1997. MR 98c:53071

[Ta 2] I. Taimanov, Surfaces of revolution in terms of solitons, Ann. Global Analysis and Geom. 15 (1997), pp. 419-435. CMP 98:04

[We] J. Weiner, On a problem of Chen, Willmore, et al., Indiana Univ. Math. J. 27 (1978), pp. 19-35. MR 57:7466

Department of Mathematics, Universidad Pais Vasco, Bilbao, Spain

E-mail address: mtpgabeo@lg.ehu.es

Department of Mathematics, Case Western Reserve University, Cleveland, Ohio 44106

E-mail address: jx16@po.cwru.edu 